International Journal of Advances in Chemistry (IJAC) Vol.2, No. 1 ,February 2016

\title{
DEVELOPMENT OF ECO-FRIENDLY FLAME RETARDANT FABRIC USING PHOSPHOROUS BASED INTUMESCENCES CHEMISTRY
}

\author{
Subhas Ghosh ${ }^{1}$ and Vikas Joshi ${ }^{2}$ \\ ${ }^{1,2}$ Textile Science Laboratory, College of Technology, Eastern Michigan University, \\ Ypsilanti,48197, Michigan, USA
}

\begin{abstract}
:
A novel flame retardant application technique was developed for cotton apparel fabric using spirocyclicpentaerythritol di (phosphoryl chloride) (SPDPC), which was further synthesized into bisdiglycolspirocyclicpentaerythritolbisphosphorate $(B S P B)$. The flame retardant agent was then attached to the fabric using a sol-gel process. The treated fabric was tested on a vertical flame tester which showed very high flame retardancy as compared to the untreated cotton. TGA analysis exhibited a slow rate of weight loss and higher ultimate degradation temperature for the FR treated sample. The FR treated sample showed modest loss in strength while retaining $86.4 \%$ of its original strength. The fabric's appearance did not alter and the change in stiffness was insignificant as a result of the FR finish.
\end{abstract}

Keywords: flame retardant, synthesis, SPDPC, TGA

\section{Introduction}

Fire retardancy (FR) is an important requirement for apparel and other furnishing textiles. Textile substrate has a high surface area and close contact with skin that created more complications in developing new chemistry. Most effective flame retardant additive and finishes developed during the period between the 1950s and the 1980s raised concerns over the toxicological and environmental consequences of using such chemicals ${ }^{1}$.

Recent research studies focused on the development of an alternative to these traditional fire retardant agents. These studies have been conducted mostly on intumescent fire retardants. Horrockset al. ${ }^{1}$ synthesized spirocyclicpentaerythritol di (phosphonyl chloride) (SPDPC) as a char former. SPDPC was applied to the Proban ${ }^{\circledR}$ treated cotton fabric, which created further higher level of char formation owing to the presence of Proban ${ }^{\circledR}$ and enhanced flame retardancy ${ }^{5}$. It is important to recognize that these intumescent finishes must be substantively attached to the textile fabric without losing required textile properties to achieve wash durability. Char formation on wool keratin and nylon was also studied using polyolphosphoryl chlorides ${ }^{6}$. Nylon 6 and 6, 6 were phosphorylated by SPDPC, cyclic 1, 3-propanediol phosphoryl chloride (CPPC) and cyclic 2, 2-diethyl-1, 3-propanediol phosphoryl chloride (CDPPC). Based on their calculations, the authors suggested that only primary amine groups at the end of the molecular chain are possible phosphorylation sites. When a wool sample was treated with SPDPC, it also improved char formation. The authors found that like Proban-treated cotton, polyamide containing active hydrogen atoms in primary amine groups at the end of the molecular chains can also be phosphorylated by the polyolphosphoryl chlorides SPDPC, CPPC, and CDPPC. Ma et al. ${ }^{2}$ synthesized phosphate-polyester co-polymer using SPDPC. The structural properties of polyester were altered as a result of introducing a new monomer into the polyester structure. The fiber's limiting oxygen index (LOI), glass-transition $\left[\mathrm{t}_{\mathrm{g}}\right]$, and solubility parameters $[\delta]$ increased with increasing SPDPC content in the copolymer. Alongi and Ciobanu ${ }^{3}$ coated cotton fabric with silica using a sol-gel process. The authors claimed higher fabric flame retardancy and thermal stability; however, the probability of an increase in fabric thickness and stiffness were not discussed. Chen et al. ${ }^{4}$ reported a flame retardant and anti-dripping finish for poly (ethylene terephthalate) fabric.

DOI: $10.5121 /$ ijac.2016.2106 
International Journal of Advances in Chemistry (IJAC) Vol.2, No. 1 ,February 2016

PET fabric was treated with poly92-hydroxy propylene spirocyclicpentaerythritol bisphosphonate (PPPBP). The authors found reduced flammability and dripping tendency of PET fabrics using a vertical burning test. Gui-Hong Chen et al. ${ }^{7}$ investigated flame retardancy of epoxy resins after treatment with bisdiglycolspirocyclicpentaerythritolbisphosphorate (BDSPBP). They found that flame retardancy of the cured epoxy resins significantly increased when BDSPBP loading was $18 \% \mathrm{w} / \mathrm{w}$. Intumescent flame retardant can be used on apparel only if the applied finish does not alter textile properties suchas strength, stiffness, and appearance. In our investigation, we synthesized SPDPC and converted it into bisdiglycolspirocyclicpentaerythritolbisphosphorate (BSPB). This flame retardant solution was attached to the cotton fabric using a sol-gel system that did not alter significantly any textile properties of the fabric.

\section{Experimental}

\subsection{Synthesis of Spirocyclicpentaerythritoldiphosphoryl chloride (SPDPC)}

SPDPC was synthesized using a modified version of the SPDPC synthesis procedure reported by Horrocks ${ }^{2}$. Pentaerythritol, 98\% pure, MP $253-258^{\circ} \mathrm{C}$ and phosphorous oxychloride, $99 \%$ pure (BP. $105^{\circ} \mathrm{C}$ ), and ethylene glycol were obtained from Sigma Aldrich, Inc., and were used as received.

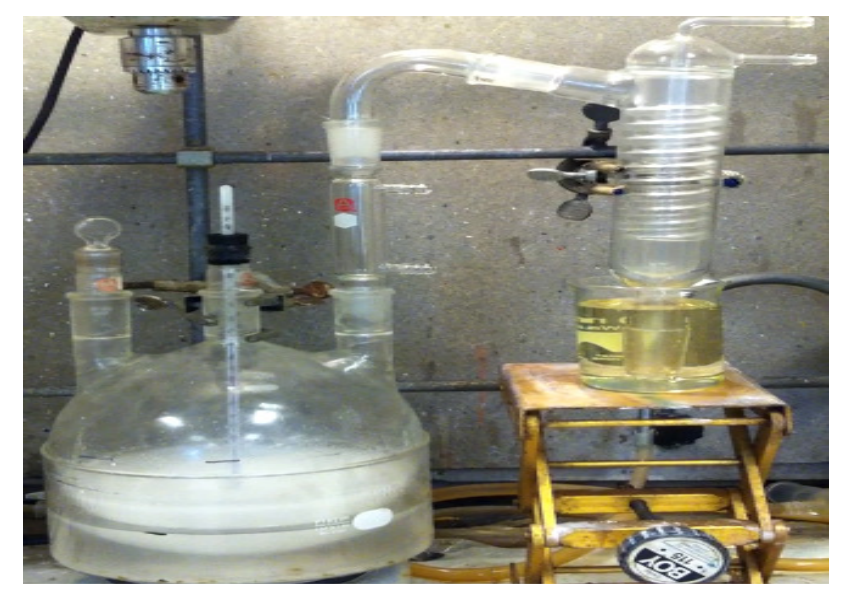

Figure 1.SPDPC synthesis and $\mathbf{H C l}$ neutralization setup.

Pentaerythritol and phosphorous oxychloride were reacted in a three-neck glass reactor at a molar ratio of $0.5: 3.5$. The reactor was connected to a magnetic stirrer, nitrogen supply, and a cold water condenser with gradual drop-by-drop addition of phosphoryl chloride to pentaerythritol. $\mathrm{HCl}$ formed during the reaction was removed into a beaker containing $\mathrm{NaOH}$ and phenolphthalein indicator as safety precaution. The temperature was slowly increased to $80{ }^{\circ} \mathrm{C}$, and reaction was continued for 2 hours. The temperature was further raised to $115^{\circ} \mathrm{C}$ and held for 20 hours. The final milky white product was washed thoroughly with tetrahydrofuran and air dried (80\% yield). Scheme 1 shows the reaction schematics for synthesis of SPDPC. 


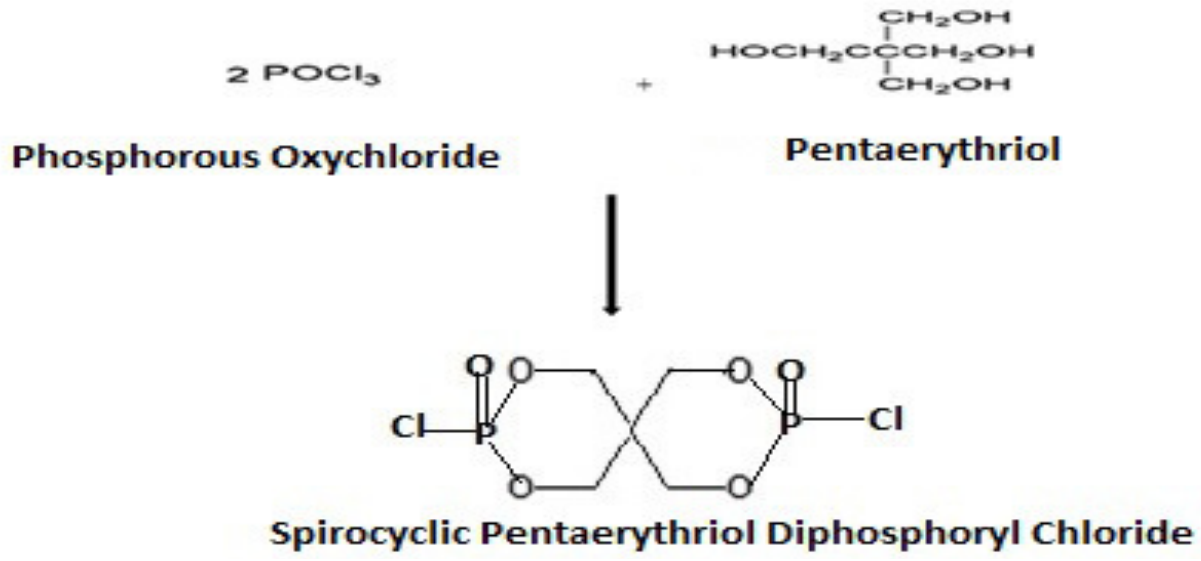

Figure 2. Reaction schematic of the formation of Spirocyclicpentaerythritoldiphosphoryl chloride (SPDPC)

\subsection{Synthesis of BisdiglycolSpirocyclicpentaerythritol bisphosphorate (BSPB)}

Ethylene glycol, $99.8 \%$ pure (B.P $195-198^{\circ} \mathrm{C}$ ), was obtained from Sigma Aldrich and was used as received. BSPB was synthesized as reported by reacting purified SPDPC with ethylene glycol at a molar ratio of $0.1: 0.25$, as shown in the Figure $3^{4}$. The reaction mixture in the threeneckreactor was heated gradually to $80{ }^{\circ} \mathrm{C}$ and held there for 6 hours. Further temperature was raised to $130{ }^{\circ} \mathrm{C}$, and the reaction mixture was held there for 4 hours. The end product was washed with diethyl ether to obtain white powder.

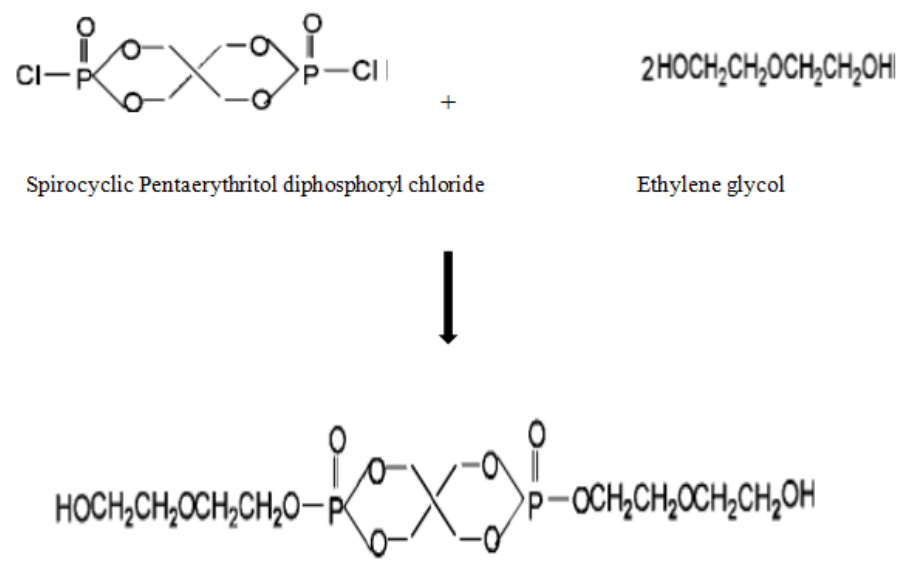

Bis-diglycol Spirocyclic pentaerythritol bisphosphorate (BSPB)

Figure3.Reaction schematic of the formation of Bis-diglycolSpirocyclicPentaerythritolBisphosphorate

(BSPB)

\subsection{Synthesis of Bis-silane and Flame retardant (FR) sol}

The synthesis of silane and sol-gel system, and its attachment to textile substrates, is the unique contribution of this research. Silquest A-Link 25 -Isocyanatopropyltriethoxysilane (B.P. 238) was obtained from Momentive and used as received. Dibutyltindilaurate reagent, acetone (99.9\% 
International Journal of Advances in Chemistry (IJAC) Vol.2, No. 1 ,February 2016

pure), and ethanol were obtained from Sigma Aldrich and used as received. Bis-silane was synthesized in two steps by reacting BSPB with triethoxysilane in the molar ratio 1:2 in presence of dibutyltindilaurate as catalyst, figure 4 . The reaction mixture is heated to $60{ }^{\circ} \mathrm{C}$ and held there for 4 hours in a three-neck glass reactor equipped with nitrogen gas, magnetic stirrer, thermometer, and a circumference condenser. Formation of bis-silane was tested using Fourier transformation infrared spectroscopy (FTIR).

The synthesized bis-silane was reacted with ethanol and water to produce a sol system. The $\mathrm{pH}$ of the reaction mixture was adjusted between 3.5 and 4 and stirred at $450 \mathrm{rpm}$, using magnetic stirrer for 15 minutes to produce a sol.
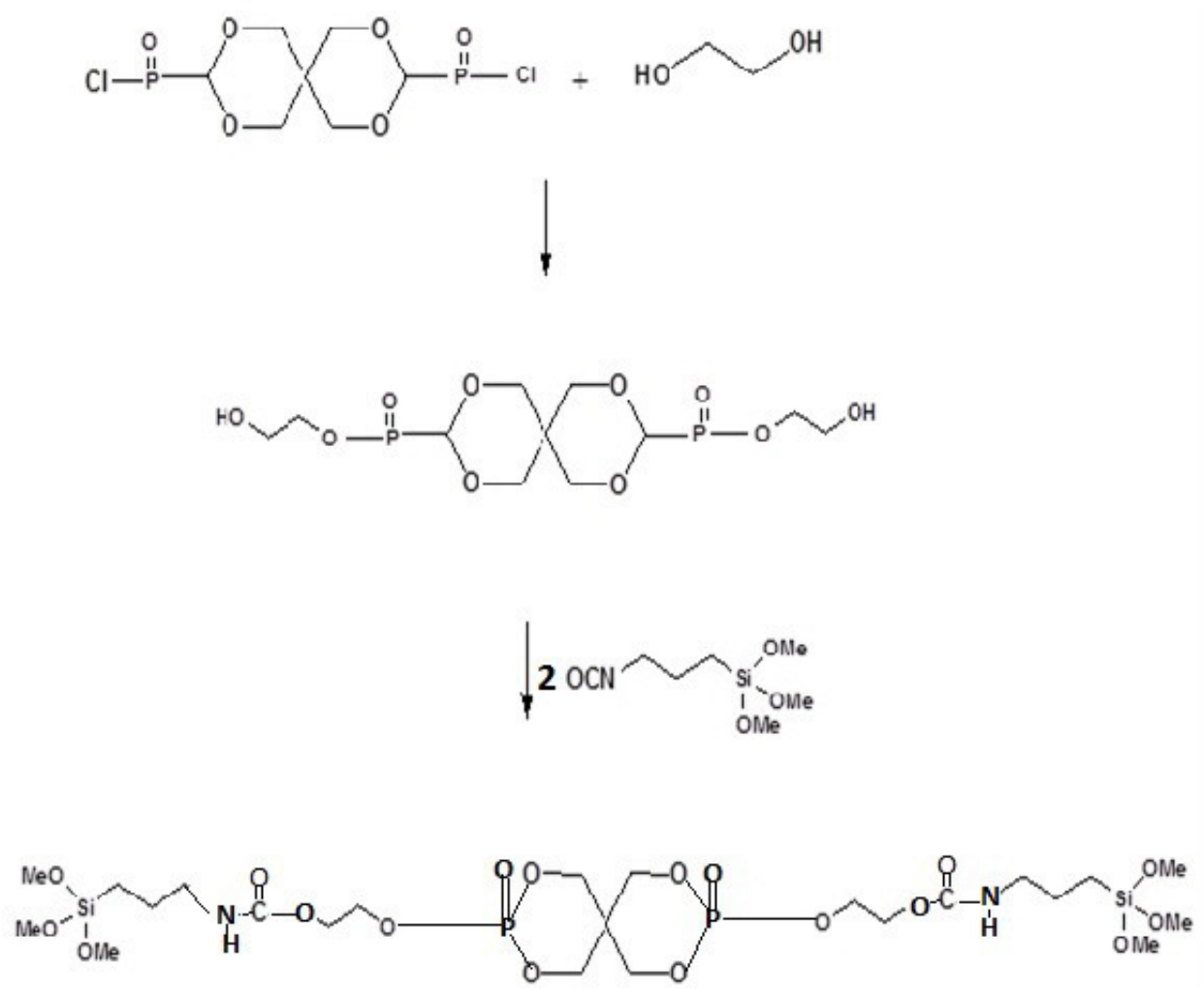

Figure 4. Reaction Schematic of formation of Bis-Silane

\subsection{Application of the FR component on textile substrate.}

The synthesized flame retardant sol was applied on a 100\% cotton fabric sample (EPI/PPI: 59/47, $3.2 \mathrm{oz} / \mathrm{sqyd}$ ). The hydroxyl group of the sol-gel flame retardant finish reacts chemically with the cellulose in cotton to form strong covalent bonds, thus attaching the flame retardant finish to cotton substrate ${ }^{3}$.The sol-gel was applied on the cotton substrate using the pad-dry-cure method. The mangle was adjusted to $25 \mathrm{psi}$, and the sample was treated with 4 dips and nips to achieve $100 \%$ wet pickup. After the application, the fabric was dried in the oven at $60{ }^{\circ} \mathrm{C}$ for 10 minutes and then cured at $115^{\circ} \mathrm{C}$ for 6 minutes in an air drying oven.

\subsection{Measurements}


International Journal of Advances in Chemistry (IJAC) Vol.2, No. 1 ,February 2016

A Bruker tensor 27 FTIR instrument was used to identify the presence of specific peaks of SPDPC, BSPB, and bis-silane.

A DSC Q200 V24.4 Build 116 differential scanning calorimeter (DSC) was used to determine the melting point of SPDPC. The sample was heated from room temperature to $300{ }^{\circ} \mathrm{C}$ at a heating rate of $10{ }^{\circ} \mathrm{C} / \mathrm{min}$.

The FR treated samples were tested on a vertical flammability tester following ASTM D 6413 procedure to observe char length after flame and afterglow of the sample.

The treated and untreated cotton samples were analyzed on a TA instrument TGA Q500 thermo gravimetric analysis instrument at a heating rate of $20{ }^{\circ} \mathrm{C} / \mathrm{min}$. The treated and untreated cotton samples" rate of "\% weight loss" was studied to draw conclusions about effectiveness of the flame retardant finish.

The tensile strength of the coated and uncoated fabric was tested on the MTS Synergie 200 CRT tensile tester by following the procedure in ASTM D5035.

The stiffness of the fabric was tested on the TABER V-5 stiffness tester by following the procedure in ASTM D5342.

\section{Results and Discussion}

\subsection{Analysis of SPDPC}

The synthesized product after reaction between pentaerythritol and phosphorous oxychloride was identified with the presence of P-Clbond at $550 \mathrm{~cm}^{-1}$ stretching. Presence of these bonds indicates the synthesis of SPDPC (Figure 2).

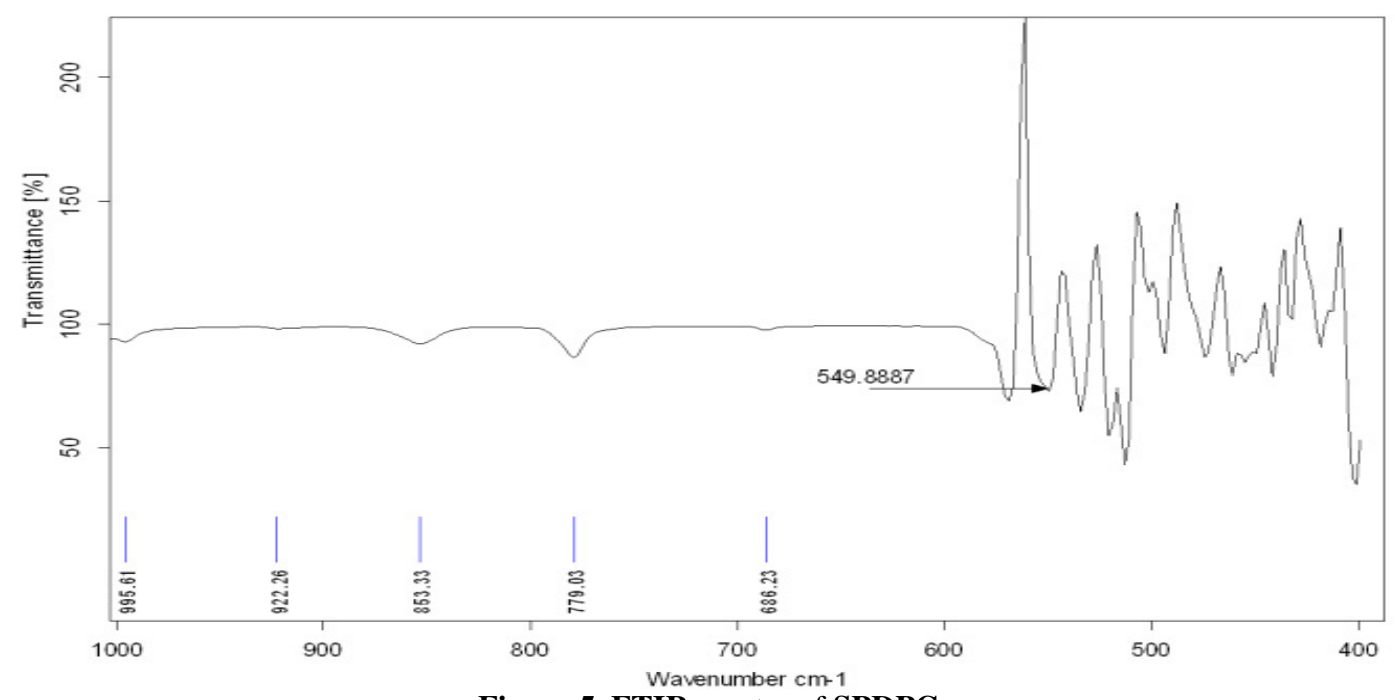

Figure 5. FTIR spectra of SPDPC

The melting point of SPDPC was found to be $245.57^{\circ} \mathrm{C}$. The results were comparable with the melting point range of SPDPC as reported in the literature ${ }^{1}$. 


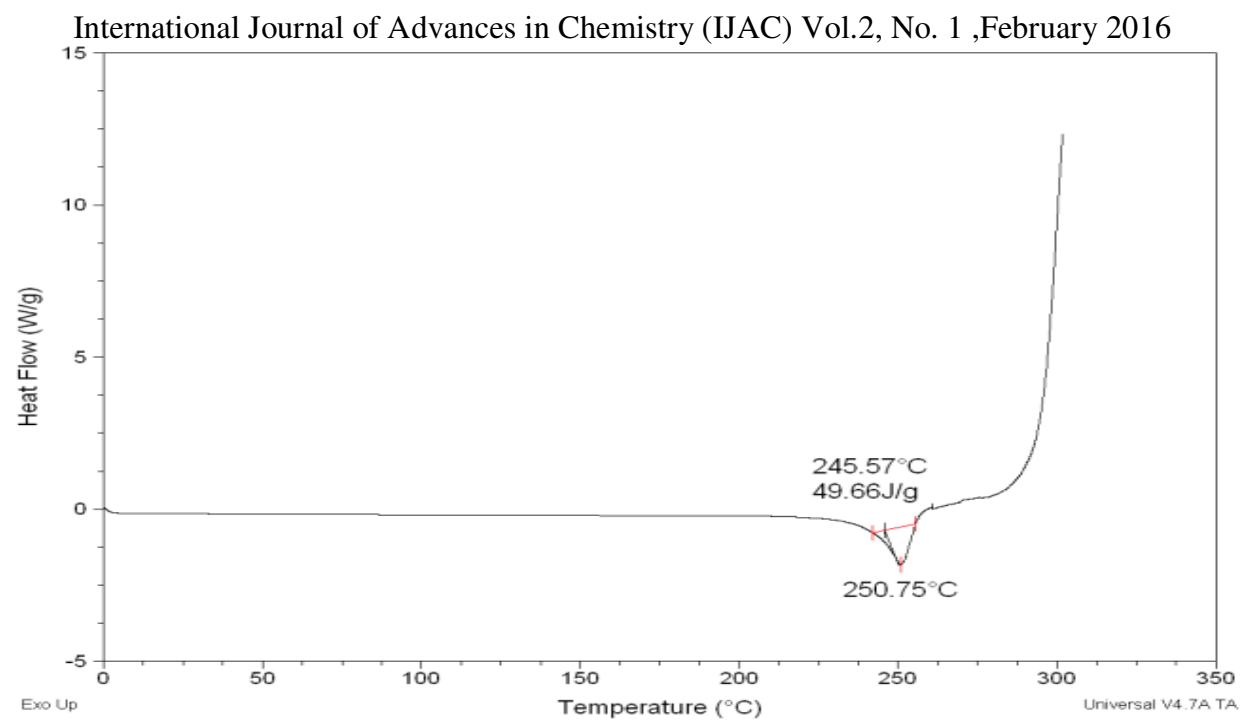

Figure 6. DSC Thermogram of SPDPC

\subsection{Analysis of BSPB}

The synthesized BSPB sample was tested for the presence of specific peaks on FTIR. The reaction between SPDPC and ethylene glycol indicates the disappearance of $\mathrm{P}-\mathrm{Cl}$ bond in process of formation of BSPB; thus the disappearance of $\mathrm{P}-\mathrm{Cl}$ stretching at $550 \mathrm{~cm}^{-1}$ confirmed the formation of BSPB.

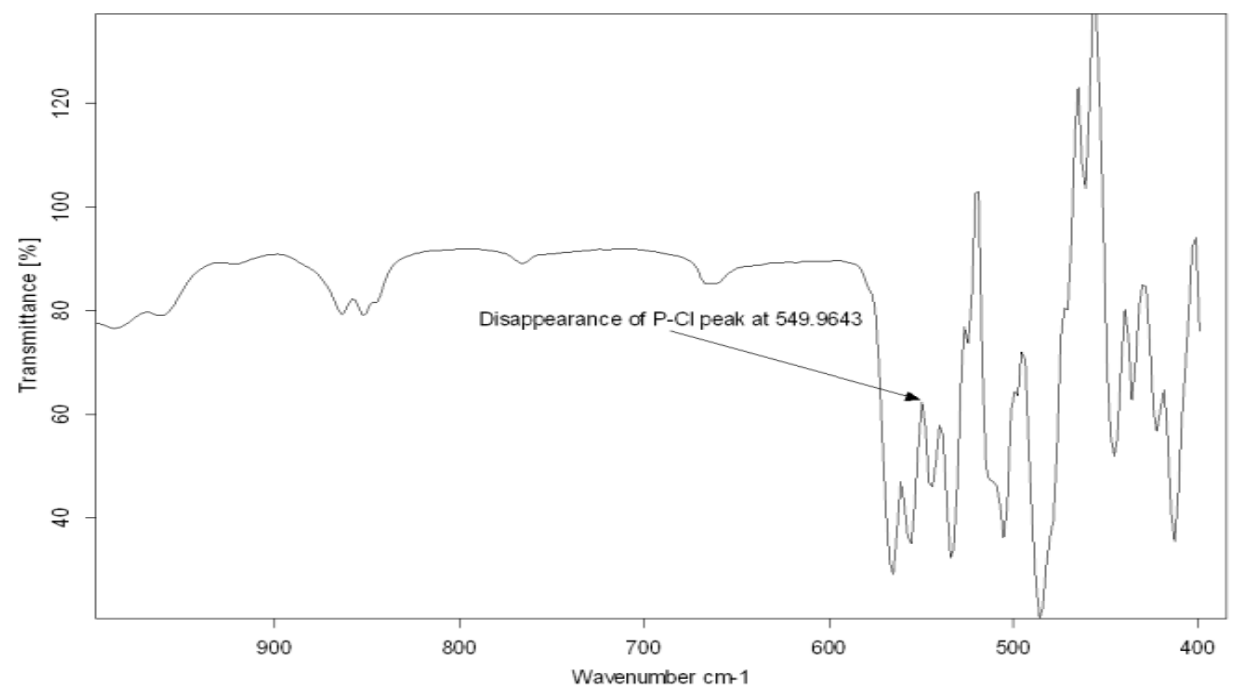

Figure 7. Illustrates the FTIR scan of BSPB 


\subsection{Analysis of Bis-silane}

The synthesized bis-silane was analyzed on FTIR to identify the peaks. Urea linkage was observed in the FTIR scan of bis-silane. The $\mathrm{C}=\mathrm{O}$ stretching and $\mathrm{N}-\mathrm{H}$ stretching can be observed on the scan at $1703 \mathrm{~cm}^{-1}$ and $1028 \mathrm{~cm}^{-1}$ arising from the presence of urea, as seen in Figure 8.

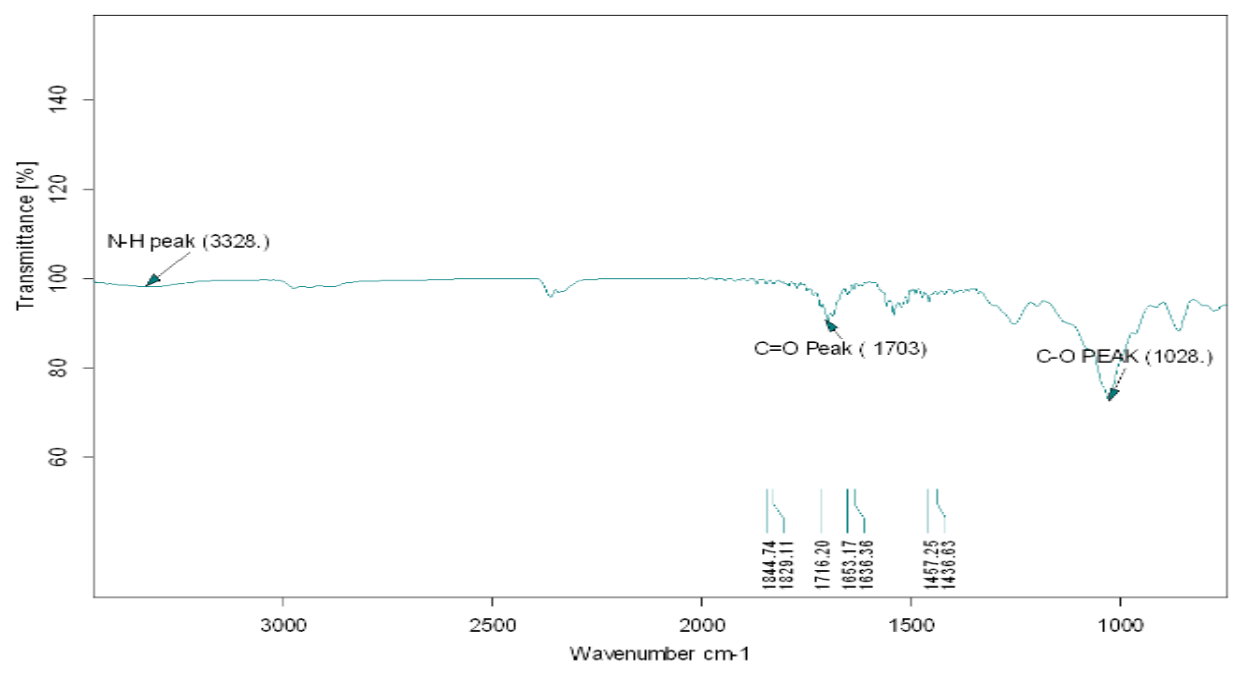

Figure 8. FTIR spectra of BisSilane

\subsection{Flame testing of the treated sample:}

During the flame testing, the treated sample self-extinguished while the flame was removed. No after flame was observed. The sample formed a one-inch char and exhibited afterglow of less than one second. The results suggest that the phosphorous-based sol gel FR treated sample passed the bench scale flame performance goals as suggested in ASTM D 6413: 2.0s, maximum after flame; $25.0 \mathrm{~s}$, maximum afterglow; and $4.0 \mathrm{in}$. maximum char length ${ }^{11}$. Therefore, the flame resistance of the cotton fabric was enhanced with the applied FR finish. However, the untreated cotton sample immediately burnt to char.
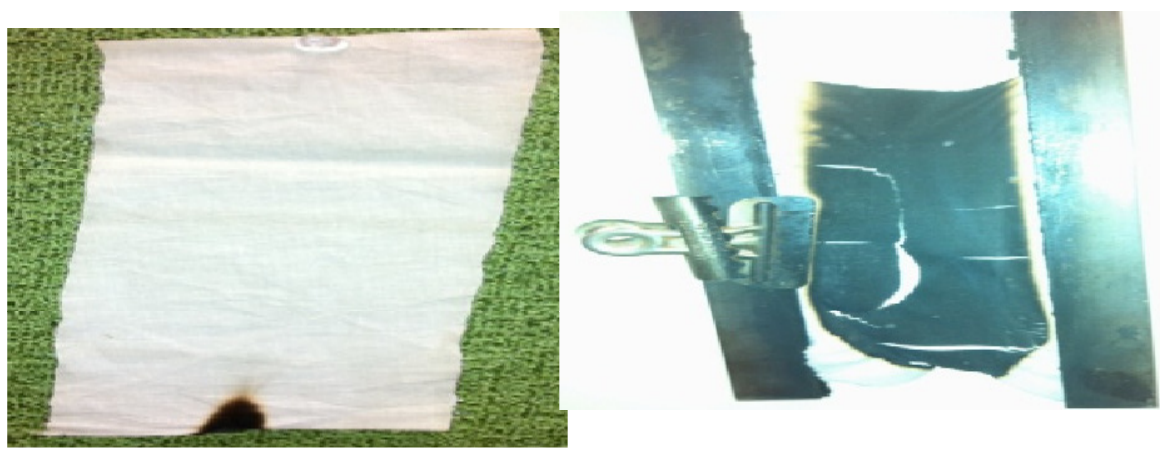

a. FR treated sample: 1-inch char lengthb. Untreated cotton sample: Burnt to char 
Figure 9.ASTM D6413vertical flammability test results (a) FR Treated sample (b) Untreated sample

\subsection{Thermo gravimetric analysis (TGA) of the treated and control cotton sample}

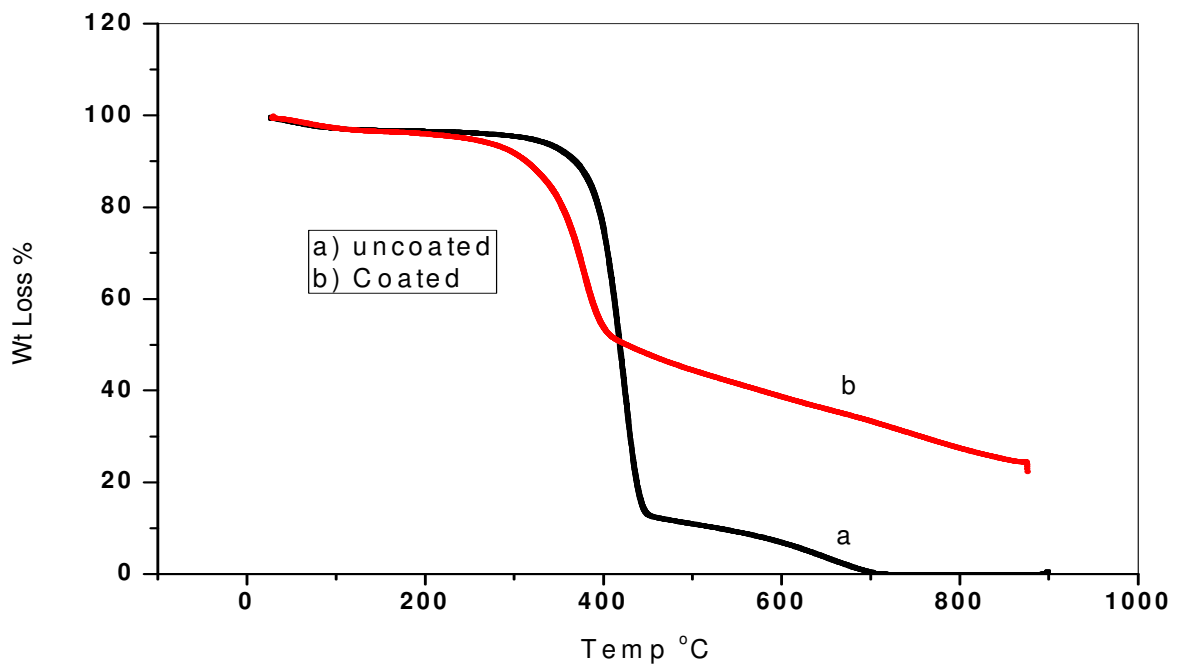

Figure 10.TGA thermogram of FR treated and untreated cotton.

The thermal degradation curve exhibited that the FR-coated sample of cotton had a slower rate of weight loss than an uncoated sample. The uncoated sample showed onset of weight loss at $380{ }^{\circ} \mathrm{C}$ and then abruptly degraded when the temperature reached $420^{\circ} \mathrm{C}$, which is the usual degradation temperature of cotton. The uncoated sample was completely degraded at about $700{ }^{\circ} \mathrm{C}$. However, the coated sample showed an onset of weight loss at $360{ }^{\circ} \mathrm{C}$ and continued gradually until $900{ }^{\circ} \mathrm{C}$, unlike abruptly degrading as the uncoated cotton sample had done. The coated sample did not degrade completely and showed about $20 \%$ weight retention at $900{ }^{\circ} \mathrm{C}$. This analysis clearly indicates that a coated flame retardant sample is thermally more stable than the uncoated cotton sample.

\subsection{Analysis of tensile and stiffness properties of the treated fabric.}

Coated and uncoated samples were tested for tensile strength and bending resistance to study the change in physical properties of the fabric due to FR coating. The tensile strength results of the coated cotton and uncoated fabric are reported in Table 1.

\begin{tabular}{|c|c|c|}
\hline Fabric & $\begin{array}{c}\text { Tensile } \\
\text { Strength } \\
\text { (lbf) ( Warp) }\end{array}$ & $\begin{array}{c}\text { Strength } \\
\text { retention \% }\end{array}$ \\
\hline Coated & 36.615 & \multirow{2}{*}{86.35} \\
\hline Uncoated & 42.405 & \\
\hline
\end{tabular}

Table 1. Tensile strength for coated and uncoated cotton fabric 
International Journal of Advances in Chemistry (IJAC) Vol.2, No. 1 ,February 2016

The coated fabric showed better strength retention after application of the FR finish than the values reported in literature, where more than $20 \%$ loss of strength was reported ${ }^{9}$. The SPDPC synthesis has high acidic $\mathrm{pH}(\mathrm{pH}<4)$. However, the acidic impurities are removed to an extent during purification of SPDPC. The FR sol system also has a slightly acidic $\mathrm{pH}, 4-4.5$. The loss in strength of cotton fabric due to FR finishing can be attributed to the acid catalyzed depolymerization of the cellulose polymer.

Stiffness of the coated and uncoated samples was measured to observe the change in stiffness of the fabric due to the FR finish.

\begin{tabular}{|c|c|c|}
\hline Fabric & $\begin{array}{c}\text { Stiffness } \\
\text { (Millinewton } \\
\text { Meters) }\end{array}$ & $\begin{array}{c}\text { Increase in } \\
\text { Stiffness } \\
\%\end{array}$ \\
\hline Coated & $2.9 \times 10^{-3}$ & \multirow{2}{*}{5.8} \\
\cline { 1 - 2 } Uncoated & $2.74 \times 10^{-3}$ & \\
\hline
\end{tabular}

Table 2 Stiffness for coated and uncoated cotton fabric.

The FR coated cotton fabric had a minor increase in stiffness of $5.8 \%$. The slight increase in stiffness of the coated fabric can be attributed to an increase in hydrogen-bonded contacts from the chemical interaction of FR sol and cellulose ${ }^{10}$. The physical testing concluded that FR coated fabric showed only a modest change in tensile strength and stiffness in comparison to the uncoated fabric. From experience, these small changes in strength and stiffness do not have a practical impact on the product. No color distortion of the fabric was observed owing to the FR finish application.

\section{Conclusion}

In this study it was possible to successfully synthesize and apply a non-toxic phosphorus-based flame resistant finish using intumescent chemistry. The FTIR and DSC analysis showed that SPDPC, BSPB, and Silane were successfully synthesized.

The flame retardant finish was applied using the pad-dry-cure method, and the coated FR cotton fabric showed an excellent flame resistance on the vertical flammability test. The TGA analysis showed that the treated fabric had a slower weight loss than the untreated cotton sample during burning. The treated cotton fabric had $86.4 \%$ tensile strength retention, and no significant change in stiffness and appearance was observed.

\section{References}

[1] Horrocks, R. A., \& Zhang, S. (2001). Enhancing polymer char formation by reaction with phosphorylated polyols.1.cellulose. Polymer, 42 (19), 8025-8033.

[2] MA, Z., Zhao, W., Liu, Y., \& Shi, J. (1997). Synthesis and properties of intumescent, phosphoruscontaining, flame-retardant polyesters. Journal of Applied Polymer Science, 63(12), 1511-1515.

[3] Alongi, J., Ciobanu, M., \&Malucelli, G. (2011). Sol-gel treatments for enhancing flame retardancy and thermal stability of cotton fabrics: optimization of the process and evaluation of the durability. Cellulose, 18(1), 167-177. 
International Journal of Advances in Chemistry (IJAC) Vol.2, No. 1 ,February 2016

[4] Chen, D., Wang, Y.-, Hu, X., Wang, D.-, Qu, M.-, \& Yang, B. (2005). Flame-retardant and antidripping effects of a novel char-forming flame retardant for the treatment of poly(ethylene terephthalate) fabrics. Polymer Degradation and Stability, 88, 349-356.

[5] Horrocks, R., \& Zhang, S. (2002). Enhancing polymer flame retardancy by reaction with phosphorylated polyols.part 2. cellulose treated with a phosphonium salt urea condensate ( proban cc) flame retardant. Fire and Materials,26, 173-182

[6] Horrocks, R. A., \& Zhang, S. (2004). Char formation in polyamides nylon 6 and nylon 66 and wool keratin phosphorylated by polyol phosphorous chlorides. Textile Research Journal, 74(5), 433-44.

[7] Chen, G. H., Yang, B., \& Wang, Y. Z. (2006). A novel flame retardant of spirocyclicpentaerythritolbisphosphorate for epoxy resins. Journal of Applied Polymer Science, 102, 4978-4982.

[8] Wilkie, C. A., Dong, M. \& Yu -Zhong, W. (2006). A novel flame retardant of Spirocyclicpentaerythriolbisphosphate for epoxy resins. Journal for Applied Polymer Science, 102, 4978-4982.

[9] Yang, C. Q., \& Yang, H. (2011). The flame retardant nomex/cotton and nylon/cotton blend fabrics for protective clothing. In Advances in modern woven fabrics technology (pp. 198-210).InTech.

[10] K, S., A, V., K, J., \& V, S. (2000). Effect of finishing agents on low stress mechanical properties of full voil fabric.Indian Journal of Fiber \& Textile Research, 25, 211-216.

[11] Winterhalter, C. A., Lomba, R. A., Tucker, D. W., \& Martin, D. O. (2005). Novel approach to soldier flame protection. Journal of ASTM International, $2(2$ 